\title{
Refining amino acid hydrophobicity for dynamics simulation of membrane proteins
}

\author{
Ronald D Hills, Jr ${ }^{\text {Corresp. } 1}$ \\ ${ }^{1}$ Department of Pharmaceutical Sciences, College of Pharmacy, University of New England, Portland, Maine, United States \\ Corresponding Author: Ronald D Hills, Jr \\ Email address: rhills@une.edu
}

Coarse-grained (CG) models have been successful in simulating the chemical properties of lipid bilayers, but accurate treatment of membrane proteins and lipid-protein molecular interactions remains a challenge. The CgProt force field, original developed with the multiscale coarse graining method, is assessed by comparing the potentials of mean force for sidechain insertion in a DOPC bilayer to results reported for atomistic molecular dynamics simulations. Reassignment of select CG sidechain sites from the apolar to polar site type was found to improve the attractive interfacial behavior of tyrosine, phenylalanine and asparagine as well as charged lysine and arginine residues. The solvation energy at membrane depths of $0,1.3$ and $1.7 \mathrm{~nm}$ correlates with experimental partition coefficients in aqueous mixtures of cyclohexane, octanol and POPC, respectively, for sidechain analogs and Wimley-White peptides. These experimental values serve as important anchor points in choosing between alternate CG models based on their observed permeation profiles, particularly for Arg, Lys and GIn residues where the all-atom OPLS solvation energy does not agree well with experiment. Available partitioning data was also used to reparameterize the representation of the peptide backbone, which needed to be made less attractive for the bilayer hydrophobic core region. The newly developed force field, CgProt 2.4, correctly predicts the global energy minimum in the potentials of mean force for insertion of the uncharged membrane-associated peptides LS3 and WALP23. CgProt will find application in studies of lipid-protein interactions and the conformational properties of diverse membrane protein systems. 


\section{Refining amino acid hydrophobicity for dynamics}

\section{2 simulation of membrane proteins}

3

4 Ronald D. Hills $\mathrm{Jr}^{*, \dagger}$

5

6 'Department of Pharmaceutical Sciences, College of Pharmacy, University of New England, 716

7 Stevens Avenue, Portland, Maine USA

8

9

10 Corresponding author

$11 *$ E-mail: rhills@une.edu 


\section{Abstract}

14 Coarse-grained (CG) models have been successful in simulating the chemical properties of

15 lipid bilayers, but accurate treatment of membrane proteins and lipid-protein molecular interactions remains a challenge. The CgProt force field, original developed with the multiscale coarse graining method, is assessed by comparing the potentials of mean force for sidechain insertion in a DOPC bilayer to results reported for atomistic molecular dynamics simulations. Reassignment of select CG sidechain sites from the apolar to polar site type was found to improve the attractive interfacial behavior of tyrosine, phenylalanine and asparagine as well as charged lysine and arginine residues. The solvation energy at membrane depths of $0,1.3$ and 1.7 $\mathrm{nm}$ correlates with experimental partition coefficients in aqueous mixtures of cyclohexane, octanol and POPC, respectively, for sidechain analogs and Wimley-White peptides. These experimental values serve as important anchor points in choosing between alternate CG models based on their observed permeation profiles, particularly for Arg, Lys and Gln residues where the all-atom OPLS solvation energy does not agree well with experiment. Available partitioning data was also used to reparameterize the representation of the peptide backbone, which needed to be made less attractive for the bilayer hydrophobic core region. The newly developed force field, CgProt 2.4, correctly predicts the global energy minimum in the potentials of mean force for

30 insertion of the uncharged membrane-associated peptides LS3 and WALP23. CgProt will find

31 application in studies of lipid-protein interactions and the conformational properties of diverse 32 membrane protein systems.

\section{Introduction}


34 Coarse-grained (CG) simulations greatly expand the timescale of events (Kmiecik et al., 2016;

35 Venable et al., 2017) that can be studied in physiologically realistic membrane systems (Ma et

36 al., 2015; Van Oosten \& Harroun, 2016). Reducing the vast number of atomic degrees of

37 freedom in the many membrane lipids, large transmembrane protein constituents, and the

38 surrounding bulk water is a necessary step that requires an accurate and transferable set of

39 interaction potentials in the reduced conformational space (Bereau et al., 2014). Construction of

40 a standalone force field for the twenty amino acids and common membrane lipids enables

41 molecular dynamics (MD) simulation of proteins of arbitrary sequence, structure and size

42 (Bereau et al., 2014; Ganesan \& Matysiak, 2014; Han et al., 2008). By enabling membrane

43 proteins to be studied in the context of their native environment, CG simulations have yielded

44 numerous insights into the nature and functional role of the dynamical interplay between proteins

45 and the lipid bilayer (Bennett \& Tieleman, 2013; Hedger \& Sansom, 2016; Marrink \& Tieleman,

46 2013; Poyry \& Vattulainen, 2016).

47 A natural method for assessing the balance of energetics in a CG model is to use umbrella

48 sampling simulations to calculate the potential of mean force (PMF) for dragging sidechain

49 analog compounds through the bilayer (MacCallum et al., 2008). Agreement with the

50 corresponding results from atomistic MD simulation has been used to assess a variety of CG

51 representations such as Martini (de Jong et al., 2013), PRIMO (Kar et al., 2014) HMMM

52 (Pogorelov et al., 2014), ELBA (Genheden \& Essex, 2015) and others (Vorobyov et al., 2016).

53 The PMFs generated from atomistic MD of sidechains such as polar glutamine and basic

54 arginine/lysine, however, have been observed to deviate from various experimental measures of

55 solvation energy. Moreover, such studies neglect the influence of both the polypeptide backbone 
56

57

58

59

60

61

62

63

and the occlusion of solvent-accessible surface area by neighboring amino acid residues in a protein chain (Singh \& Tieleman, 2011; Wimley et al., 1996).

Experimental measures for the hydrophobicity of isolated sidechains involve the partitioning of analog compounds (methane for Ala, propane for Val, etc.) in cyclohexane-water and octanol-water mixtures (Radzicka \& Wolfenden, 1988). To estimate the solvation energy for residues occluded by moderate-sized neighbor residues in the peptide chain, the octanol-to-water partitioning of Ac-WL-X-LL pentapeptides was determined (Wimley et al., 1996) relative to AcWL-G-LL (see $\Delta G^{\text {cor }}$ in Table 2 of Wimley et al.). Combining these values with the estimated $1.15 \mathrm{kcal} / \mathrm{mol}$ backbone penalty for nonpolar solvation of the glycyl $-\mathrm{CH}_{2} \mathrm{CONH}-$ unit yielded a whole-residue hydrophobicity scale that is useful for predicting transmembrane regions in proteins (White \& Wimley, 1998). For a measure of exposed sidechains, solvation energies were corrected for occlusion by the host peptide using measurements of the nonpolar solventaccessible surface area as compared to Ac-GG-X-GG (see $\Delta G^{\mathrm{GXG}}$ in Table 2 of Wimley et al.). Another measure for the fully exposed residue is the octanol-water solvation energy of an acetyl amino acid amide dipeptide: Ac-X-amide (Fauchere \& Pliska, 1983). Experimental values are compiled in the Supplemental Information (Table S1, CSV format).

In the present work, parameter modifications of the CgProt force field (Hills Jr et al., 2010; Ward et al., 2012) are explored by comparing their resulting sidechain permeation profiles in a DOPC bilayer. By reassigning the CG site type definitions for select sidechain interaction centers, the permeation profiles of several amino acids are found to improve relative to either atomistic simulation results or the known experimental solvation energies. The sidechain parameters are then coupled to an improved description of the peptide backbone. The resulting force field, CgProt version 2.4, is assessed for the ability of designed helical peptides to remain 
79 inserted in the membrane during microsecond MD simulations. While the previous version,

80 CgProt 2.3, relied on terminal charges to anchor transmembrane peptides across the bilayer, the

81 current force field captures the proper orientation of neutral peptides LS3 and WALP23 in the

82 bilayer, consistent with experiments using capped uncharged termini. The refinement of amino

83 acid hydrophobicity makes CgProt a powerful tool for studying lipid-protein interactions.

\section{Methods}

\section{Coarse grain model}

86 The first iteration of the CgProt force field was developed for aqueous proteins using the

87 multiscale coarse graining (MS-CG) method (Hills Jr et al., 2010). MS-CG is a variational force-

88 matching procedure for developing a self-consistent set of $\mathrm{CG}$ interaction potentials given a

89 reference ensemble generated from atomistic MD (Noid et al., 2008). A strength of MS-CG is

90 that it can map an inherently many-body PMF into effective pairwise interactions without an $a$

91 priori assumed functional form (Noid et al., 2007). To construct a protein force field, tabulated

92 nonbond potentials were developed for five unique CG site types ca/p/ap/pos/neg assigned to

93 groups of backbone and sidechain atoms based on atomistic reference simulations of peptide

94 unfolding in water. Because the model detail emphasized the amino acid sidechains rather than

95 backbone, CgProt itself is not useful for de novo folding of proteins. When deployed with an

96 elastic network encoding the backbone native conformation, the force field can effectively

97 simulate protein conformational transitions (Ward et al., 2012).

98 To enable the simulation of membrane proteins, atomistic simulations were performed of

99 peptide unfolding in aqueous mixtures of phospholipids. MS-CG was then used to develop lipid-

100 protein interaction potentials for the headgroup and tail sites: $\mathrm{ch} / \mathrm{hh} / \mathrm{ph} / \mathrm{gl} / \mathrm{e} 1 / \mathrm{e} 2 / \mathrm{s} 1 / \mathrm{sd} / \mathrm{sm}$ for 
101 common physiological lipids (Hills Jr \& McGlinchey, 2016; Ward et al., 2012). For the lipid-

102 lipid interactions needed to construct a membrane model, parameters were adapted from a

103 previous MS-CG model developed for a DOPC:DOPE bilayer in the absence of explicit water

104 molecules $(L u \&$ Voth, 2009). The present work assesses the physical realism that can be

105 obtained by combining the two protein and lipid force fields.

106 The polypeptide is represented as a chain of backbone beads defined at the alpha carbon

107 positions with up to four CG sites defined within each sidechain as the mass centers of non-

108 overlapping subgroups of atoms. In contrast to approximations made in other CG models in

109 order to obtain larger time steps, Boltzmann inversion is used to assign accurate harmonic

110 interaction potentials that reproduce the CG bond fluctuations observed in atomistic MD (Hills

$111 J r, 2014)$. Fourth order polynomials were used to fit the 3-body angle distributions observed for

112 each of the 20 amino acids (Hills $J r$ et al., 2010). The C $\alpha$ backbone is assigned generic but

113 sequence-dependent torsional potentials that allow for the adoption of either $\alpha$-helix or $\beta$-sheet

114 secondary structure (Karanicolas \& Brooks III, 2002). The original CgProt parameterization

115 employed a unique site type for the backbone $\mathrm{C} \alpha$ bead: $c a$. To reproduce the bilayer permeation

116 profiles of backbone moieties, version 2.4 assigns the existing apolar site type (ap) to Ala

117 residues and the existing polar site (p) type to all other backbone beads (Fig. 1). New site type

118 assignments are explored for select sidechain $p$ or $a p$ sites, but the original nonbond potentials

119 tabulated for each site type pair combination were not modified.

120 Given the lack of explicit water, dynamics employ the stochastic Langevin dynamics

121 routine with a damping friction coefficient of $\gamma=0.5 \mathrm{ps}^{-1}$, a significant speedup relative to

122 protein dynamics in real water (Cerutti et al., 2008). The lateral diffusion of lipids in the bilayer

123 is enhanced 30-fold in CgProt (Fosso-Tande et al., 2017). An effective temperature of $k T=2.24$ 
$124 \mathrm{~kJ} / \mathrm{mol}$ is used in the present work as it has been shown to reproduce lipid bilayer structure

125 across different liquid phase systems (Hills $J r \&$ McGlinchey, 2016). Nonbond interactions have

126 a $1.2 \mathrm{~nm}$ cutoff and the neighbor list is updated every step. As recommended by others (Arnarez

127 et al., 2015), the implicit solvent bilayer simulations were performed in the fixed volume NVT

128 ensemble. The Gromacs 4.6.7 simulation package was used (Pall et al., 2015) with a 5 fs

129 integration time step.

\section{Sidechain insertion PMFs}

$131 \mathrm{CgProt}$ is assessed and refined by comparing the energetics of sidechain bilayer insertion to

132 PMFs from atomistic simulations reported in the literature (de Jong et al., 2013; Johansson \&

133 Lindahl, 2008; MacCallum et al., 2008). Umbrella sampling calculations were performed with a

134 harmonic force constant of $350 \mathrm{~kJ} \mathrm{~mol}^{-1} \mathrm{~nm}^{-2}$ applied between the mass centers of the sidechain

135 and lipid bilayer in the $z$-dimension. As in previous atomistic studies (MacCallum et al., 2008),

136 an additional copy of the sidechain was placed $3.7 \mathrm{~nm}$ above the first in order to duplicate the

137 number of data collected in a single run. A total of 21 umbrella windows with $0.2 \mathrm{~nm}$ spacing

138 were simulated for $50 \mathrm{~ns}$ each. To ensure overlap between successive windows, sidechain 1 was

139 restrained at depths spanning $z=-3.3$ to $z=+0.5 \mathrm{~nm}$ from the bilayer center, while sidechain 2

140 spanned $z=0$ to $4 \mathrm{~nm}$. The two halves of the PMFs were computed using g_wham (Hub et al.,

1412013 ) in Gromacs and treated as independent samples to determine the largest error over the 400

142 bins. After shifting to zero in bulk water, the maximum PMF error for most sidechains was

143 between 0.1 and $0.5 \mathrm{~kJ} / \mathrm{mol}$ at the bilayer center $(z=0)$. Exceptions were Arg, Lys and Asp,

144 whose strong repulsion at $z=0$ had a maximum error of 2.0-2.6 $\mathrm{kJ}$ even after increasing the

145 simulation length to $250 \mathrm{~ns}$. 
A large periodic simulation box (256 DOPC molecules, $9.4 \mathrm{~nm}$ box edge) was used to

147 minimize boundary effects in the umbrella simulations (Neale \& Pomes, 2016; Nitschke et al.,

148 2016). By comparison, a $250 \mathrm{~ns}$ simulation performed with a small box (64 DOPC, $4.7 \times 4.7 \times 9$

$149 \mathrm{~nm}$ ) containing two arginine sidechains reveals that the small simulation significantly

150 overestimates the steepest part of the PMF at a distance of $z=1 \mathrm{~nm}$ from the bilayer center. The

151 larger box also decreases the likelihood that the two sidechain copies influence each other. A

152 comparison PMF obtained from a simulation with only one arginine sidechain in a $9.4 \mathrm{~nm}$ box

153 fell within the $2.6 \mathrm{~kJ}$ margin of error of the two sidechain simulation.

\section{Peptide insertion simulations}

155 The membrane binding or insertion behavior of synthetic peptides in the DOPC bilayer was used

156 to test the balance of membrane-protein interactions in CgProt, as has been performed with other

157 force fields (Bereau et al., 2015; Bereau \& Kremer, 2016; Bereau et al., 2014; Bond et al.,

158 2007; Hall et al., 2011; Kar et al., 2014; Pulawski et al., 2016; Ward et al., 2017). The sequence

$159 \mathrm{GWW}(\mathrm{LA})_{8} \mathrm{LWWA}$ (WALP23) of the WALP $n$ series of single-pass transmembrane peptides

160 was simulated since its $25.5 \AA$ hydrophobic length (Kim \& Im, 2010) nearly matches the

161 hydrophobic thickness of the DOPC bilayer: $29 \AA$ as measured by the mean distance between

162 ester sites. The highly charged transmembrane peptides $\mathrm{K}_{2} \mathrm{~L}_{24} \mathrm{~K}_{2}$ and $\mathrm{K}_{2}(\mathrm{LA})_{12} \mathrm{~K}_{2}$, referred to as

$163 \mathrm{~L}_{24}$ and $(\mathrm{LA})_{12}$, respectively, were also assessed for their bilayer insertion stability (Liu et al.,

164 2004; Zhang et al., 1995). Last, the designed amphipathic peptide (LSSLLSL) 3 , referred to as

165 LS3, was tested for its binding at the membrane-water interface (Lear et al., 1988).

166 In versions 2.3 and earlier CgProt relied on terminal charges to anchor peptides within

167 the membrane (Fosso-Tande et al., 2017). In this work, each N- and C-terminus is kept neutral in

168 order to rigorously assess the insertion behavior of the current force field and its new backbone 
169 description. This is consistent with experimental studies (Holt et al., 2009; Zhang et al., 1995) of

170 the designed peptides in which the N- and C-termini are capped with acetyl and amide groups,

171 respectively. Each of eight simulation runs were conducted for $2 \mu \mathrm{s}$ in a $9.5 \times 9.5 \times 10 \mathrm{~nm}$ box

172 containing 256 DOPC molecules, with the peptide starting in either a membrane-bound or

173 completely desorbed state. Peptides were restrained in an ideal right-handed $\alpha$-helix

174 conformation by applying a harmonic GROMOS-96 angle $\left(\theta_{0}=89^{\circ}, k_{\theta}=2000 \mathrm{~kJ} \mathrm{~mol}^{-1}\right)$

175 between each successive $\mathrm{C} \alpha-\mathrm{C} \alpha-\mathrm{C} \alpha$ and a harmonic torsion $\left(\varphi_{0}=50^{\circ}, k_{\varphi}=2000 \mathrm{~kJ} \mathrm{~mol}^{-1} \mathrm{rad}^{-2}\right)$

176 for $\mathrm{C} \alpha-\mathrm{C} \alpha-\mathrm{C} \alpha-\mathrm{C} \alpha$ terms. The angle of peptide insertion with respect to the bilayer normal was

177 calculated using the vector spanning alpha carbons 5 and 16 for LS3, 5 and 19 for WALP23, and

1788 and 26 for $\mathrm{L}_{24} /(\mathrm{LA})_{12}$.

\section{Peptide insertion PMFs}

180 The PMFs for the insertion of LS3 and WALP23 peptides in a DOPC bilayer were determined

181 using umbrella sampling simulations (Bereau et al., 2015; Bereau \& Kremer, 2016; Ward et al.,

182 2017). Umbrella windows incorporated a single peptide placed at $0.2 \mathrm{~nm}$ intervals along the $\mathrm{z}$ -

183 axis. Starting structures were generated by pulling the peptide into the bilayer over a $4.6 \mathrm{~ns}$

184 simulation $\left(2 \times 10^{4} \mathrm{~kJ} \mathrm{~mol}^{-1} \mathrm{~nm}^{-2}\right.$ force constant $)$ starting from either side of the bilayer at $z=$

$185 \pm 4.6 \mathrm{~nm}$. For each peptide, a total of forty-eight windows were simulated for $100 \mathrm{~ns}$ each using a

186 force constant of $650 \mathrm{~kJ} \mathrm{~mol}^{-1} \mathrm{~nm}^{-2}$ force constant for the peptide center of mass relative to the

187 bilayer normal. Snapshots were recorded every 1 ps. The first 16 ns of simulation time was

188 excluded from the WHAM calculation to allow for the peptide orientation to equilibrate.

189 Results and Discussion

190 Sidechain permeation profiles 
191 The DOPC bilayer can be divided into four regions (Fig. 2) to aid in comparing to previous work

192 (Genheden \& Essex, 2015; MacCallum et al., 2008). The tail region, found within $1 \mathrm{~nm}$ of the

193 bilayer center, contains only hydrophobic tails. The ester region from $z=1$ to $1.8 \mathrm{~nm}$ begins at

194 the minimum depth of the carbonyls, has a falling lipid tail density, and contains most of the

195 glycerol density. The head group region from 1.8 to $2.8 \mathrm{~nm}$ contains the largest portion of the

196 phosphate and choline head group density. The fourth region represents the pure water phase.

197 These trends in DOPC lipid partial densities are in agreement with atomistic MD simulations

198 (MacCallum et al., 2008).

199 PMF insertion profiles are reported for each sidechain from $z=0$ to $3.7 \mathrm{~nm}$ (Fig. 3). For

200 sidechains in the original force field that did not exhibit expected behavior, alternate CG

201 descriptions were explored. Note that Cys and Pro sidechains consisting of a lone apolar bead are

202 indistinguishable in absence of the $\mathrm{C} \alpha$ site, as are polar Ser and Thr. The C $\beta-\mathrm{C} \gamma$ virtual bond

203 between apolar CG sites is nearly identical in Ile and Met and leads to PMF profiles that are

204 indistinguishable. The single polar site for Ser/Thr has a $4.4 \mathrm{~kJ} / \mathrm{mol}$ attractive well at $z=1.7 \mathrm{~nm}$

205 and a $10 \mathrm{~kJ}$ barrier to crossing the bilayer center at $z=0$. This compares well to the atomistic

206 PMFs obtained in OPLS for Ser and Thr, which have 1-4 kJ attraction at $z=1.5 \mathrm{~nm}$ and a

207 repulsive barrier for $z<1 \mathrm{~nm}$ that reaches $15 \mathrm{~kJ}$ (MacCallum et al., 2008). For Cys, the single

208 apolar site has an $8.5 \mathrm{~kJ}$ repulsion at $z=2.1 \mathrm{~nm}$ and is attractive by $4-6.6 \mathrm{~kJ}$ for $z<1 \mathrm{~nm}$. This

209 corresponds well with the atomistic PMF reported by MacCallum et al. for cysteine: $3 \mathrm{~kJ}$

210 repulsion at $\mathrm{z}=2.1 \mathrm{~nm}$ and $4-7 \mathrm{~kJ}$ attraction for $z<1.5 \mathrm{~nm}$. In contrast, the orginal CgProt force

211 field (v.1) employed a polar site for cysteine, overestimating the energy of insertion into the tail

212 region by $13 \mathrm{~kJ} / \mathrm{mol}$. For Val, a second apolar site was added to its $\mathrm{CG}$ description to capture the 
213 hydrophobic nature of the atomistic PMF for valine: $5 \mathrm{~kJ}$ repulsion at $\mathrm{z}=2 \mathrm{~nm}$ and $>10 \mathrm{~kJ}$

214 attraction for $z<1.5 \mathrm{~nm}$.

215 Ile and Leu also contain two apolar sites, having a 17-22 kJ attraction for the tail region

216 of the bilayer. The short $1.6 \AA \mathrm{C} \beta-\mathrm{C} \gamma$ bond of Leu and Val allows them to pack more favorably

217 inside the tail region than Met and Ile (2.5 $\AA$ bond). The PMFs are in qualitative agreement with

218 the $15-22 \mathrm{~kJ}$ attraction for $z<1 \mathrm{~nm}$ in the Ile and Leu atomistic profiles (MacCallum et al.,

219 2008), but there is a larger repulsion in the head group region. The three apolar sites in the

220 original description for Phe overestimate its atomistic PMF, which bears a $15 \mathrm{~kJ}$ attraction for $z<$

$2211.5 \mathrm{~nm}$ (de Jong et al., 2013; MacCallum et al., 2008). Better agreement for aromatic

222 phenylalanine was found by assigning the polar site type to $\mathrm{C} \delta$. Neutral histidine contains one

223 apolar and two polar sites in CgProt with a $14 \mathrm{~kJ}$ attraction at $z=1.5 \mathrm{~nm}$ and $8 \mathrm{~kJ}$ repulsion at $z=$

224 0. The atomistic PMF for His is similar in these features but has a smaller energy barrier in the

225 vicinity of $z=2.5 \mathrm{~nm}$ (de Jong et al., 2013). Using three polar sites for His reduces the barrier

226 but results in too strong an attraction for the head group region.

227

The atomistic PMFs for Trp and Tyr are 21 and $13 \mathrm{~kJ}$ attractive at $\mathrm{z}=1.3 \mathrm{~nm}$ and rise to

$228-5$ and $+7 \mathrm{~kJ}$ at $z=0$, respectively (MacCallum et al., 2008). The best agreement for tryptophan

229 in $\mathrm{CgProt}$ is to assign three apolar sidechain sites along with one polar site at $\mathrm{C} \gamma$. For tyrosine,

230 assignment of polar sites at $\mathrm{C} \gamma$ and $\mathrm{C} \varepsilon$ in the current version improves the permeation profile in

231 the bilayer tail region. A significant energy barrier is found in both potentials at $z=2.5 \mathrm{~nm}$. As

232 this falls outside the lipid density of the bilayer (Fig. 2), the barrier is likely to affect the kinetics

233 of insertion but not the thermodynamic stabilization for proteins residing in the membrane.

234 The atomistic PMFs for Gln and Asn are 9 and $7 \mathrm{~kJ}$ attractive at $z=1.5 \mathrm{~nm}$ and 20 and 24

$235 \mathrm{~kJ}$ repulsive at $z=0$, respectively (de Jong et al., 2013; MacCallum et al., 2008). To reproduce 
236 this behavior, the $\mathrm{C} \beta$ site type of glutamine was changed from apolar to polar, resulting in

237 stronger attraction at the membrane interface and stronger repulsion in the hydrophobic tail

238 region. For asparagine, the best agreement was obtained by removing its apolar site altogether,

239 which involved increasing the $\mathrm{C} \alpha-\mathrm{C} \beta$ bond from 2.0 to $2.6 \AA$. Glu and Asp are the only

240 sidechains with a monotonic repulsion observed in atomistic MD for all regions in the bilayer,

241 along with an $80 \mathrm{~kJ}$ barrier to crossing the bilayer in their charged form. CgProt reproduces the

242 strong repulsion in the tail and ester regions using one polar and one negative site type. In place

243 of a screened Coulomb potential, the force field energy function successfully combines

244 electrostatic and van der Waals forces into a single tabulated function for each of the positive and

245 negative site types ( $L u \&$ Voth, 2009; Noid et al., 2008).

246 The atomistic PMFs for $\mathrm{Arg}^{+}$and $\mathrm{Lys}^{+}$are $+50 \mathrm{~kJ}$ repulsive at $z=0$ but have a large

247 attractive well spanning $z=0.8$ to $2.5 \mathrm{~nm}$ with a minimum of $-20 \mathrm{~kJ} / \mathrm{mol}$ (de Jong et al., 2013;

248 MacCallum et al., 2008). Arginine and lysine were too repulsive for the membrane in CgProt

249 v.1. The solution employed in CgProt 2.4 is to reassign their apolar $\mathrm{C} \beta$ sites to be polar. An

250 alternate sidechain description was also tested consisting of two polar sites and a positive site

251 connected by short $1.5 \AA$ bonds (p.p.pos). Experimental measures of hydrophobicity were used

252 to discriminate between alternate $\mathrm{CG}$ descriptions, which are discussed in the next section.

253 There has been much interest in the attraction of positive-charged sidechains for the lipid

254 bilayer (Gumbart \& Roux, 2012; MacCallum \& Tieleman, 2011; Sun et al., 2015). Findings are

255 now emerging as to its potential functional relevance for peptides (Gleason et al., 2013; Hu et

256 al., 2014; MacCallum et al., 2011; Nakao et al., 2016; Rice \& Wereszczynski, 2017). The

257 favorable attraction of basic residues for the ester bilayer region has been dubbed snorkeling. A

258 bilayer defect is created in which polar lipid head groups dive down to solvate the positive 
259 charge. A representative snapshot from the CgProt umbrella simulations (Fig. 4) shows the

260 arginine sidechain near the center of the bilayer producing local changes in the depth of glycerol,

261 phosphate and choline groups, similar to results observed in atomistic MD (MacCallum et al.,

262 2008).

\section{Experimental partition energies}

264 Experimental values for the partitioning of sidechain analog compounds from water to

265 cyclohexane were correlated with the free energy of transfer to the bilayer center at $z=0$

266 (MacCallum et al., 2008; Pogorelov et al., 2014; Vorobyov et al., 2016) computed from the

267 CgProt 2.4 PMFs. A linear regression was performed for all sidechains except charged Asp, Glu

268 and Lys. Since the experiments did not control the ionization state (Radzicka \& Wolfenden,

269 1988), the Asp, Glu and Lys sidechains are expected to be neutral in the nonpolar environment of

270 cyclohexane based on analysis of their $\mathrm{pKa}$ values and solvation energies from atomistic MD

271 (MacCallum et al., 2008). A strong correlation (Pearson coefficient of $r=0.95$ ) is observed

272 between CgProt 2.4 and experiment (Fig. 5A), similar to the correlation between cyclohexane

273 solvation energies and the atomistic PMF values of MacCallum et al. (Table 1).

274 The transfer free energy of sidechain analogs from water to octanol was measured in the

275 same study (Radzicka \& Wolfenden, 1988). Positing that octanol has properties analogous to the

276 ester region of the bilayer, we correlated the octanol solvation energies to the PMF values of

277 MacCallum et al. for transferring solute to a membrane depth of $z=1.3 \mathrm{~nm}$ and obtained a good

278 correlation of $r=0.92$ upon exclusion of outliers Arg and Lys (Table 1). For the PMFs calculated

279 in CgProt 2.4, a correlation of $r=0.78$ was found upon exclusion of sidechains Asp, Glu and Lys

280 (Fig. 5B). Arginine, which is predicted to be protonated in the bilayer ester region (MacCallum

281 et al., 2008), falls on the regression line. 

atomistic PMFs, excluding outliers Arg and Lys. Similarly, a prominent linear correlation (r =

et al. controlled for protonation state, allowing for comparison to all charged sidechain in a glycine peptide $(r=0.91)$.

To compare the partitioning of sidechains to the polar environment of the bilayer interface, we take the whole-residue solvation energies derived from the WLXLL peptide series in POPC and subtract the $+0.04 \mathrm{~kJ} / \mathrm{mol}$ value for glycine (Wimley \& White, 1996). Using the free energy at a depth of $z=1.7 \mathrm{~nm}$, a good correlation of $r=0.86$ is obtained for the atomistic PMFs of MacCallum et al., excluding outliers Arg and Lys. For CgProt, a weak correlation of $r=0.51$ is observed. His, Arg and Gln were found to be outliers and were excluded from the linear regression (Fig. 5D). The various transfer energies for cyclohexane, octanol, and POPC measurements are plotted at $z=0,1.3$, and $1.7 \mathrm{~nm}$, respectively, in Figure 3 . Together the experimental data points serve as a guide for selecting between alternate CG descriptions. This is particularly useful for Arg and Lys, for which more positive solvation energies are measured in experiments as compared to atomistic MD.

\section{Peptide backbone parameters}

302 Earlier versions of CgProt enabled the rapid insertion of peptides with charged N- and C-termini

303 in unbiased MD simulations (Fosso-Tande et al., 2017). This was due to the attractive nature of

304 the unique backbone type $(c a)$ for the bilayer core. PMFs are calculated for the insertion of one 
305 and two-residue ca backbone units in the bilayer, revealing a free energy minimum at $z=0.7 \mathrm{~nm}$

306 of -16.5 and $-35 \mathrm{~kJ} / \mathrm{mol}$, respectively (Fig. 6). This is in stark contrast with the $+1.15 \mathrm{kcal} / \mathrm{mol}$

307 water to octanol transfer energy for the glycyl $-\mathrm{CH}_{2} \mathrm{CONH}-$ unit (Wimley et al., 1996). PMFs

308 calculated for one and two-residue backbone units using the polar site type ( $p)$ are in better

309 agreement with PMFs obtained from atomistic MD for acetamide and other backbone mimics

310 (Bereau \& Kremer, 2016; Sandoval-Perez et al., 2017), as well as the positive partition energies

311 obtained from experiment. For the CgProt representation of alanine, the backbone bead is the

312 sole CG site. The PMF of methane $\left(\mathrm{CH}_{4}\right)$ has a $-8.4 \mathrm{~kJ} / \mathrm{mol}$ free energy minimum at $z=0$ and its

313 cyclohexane partition energy is $-7.6 \mathrm{~kJ} / \mathrm{mol}$ (Radzicka \& Wolfenden, 1988). Alanine residues are

314 therefore represented using the apolar site type (ap), which provides good agreement with the

315 Wimley-White whole-residue values (Fig. 6).

316 Peptide insertion properties

317 Simulations of peptide insertion were used to test the balance of backbone and sidechain

318 interactions in CgProt 2.4 (Bereau \& Kremer, 2016). Multiple independent simulations were

319 performed for the designed helical peptides LS3, WALP23, $\mathrm{L}_{24}$ and (LA) 12 starting from either

320 membrane-bound or desorbed states. In each simulation, the peptide adopted a single stable

321 orientation within the membrane, with the exception of the desorbed WALP23 simulation, in

322 which the peptide did not adsorb onto the membrane. The kinetic barrier to WALP23 insertion

323 can be explained by the barrier in the PMF for tryptophan at the membrane interface near $z=2.5$

$324 \mathrm{~nm}$ (Fig. 3). The orientation of the helix axis relative to the bilayer normal is shown for each

325 simulation in Table 2, along with the membrane depth of the peptide center of mass. The final

326 equilibrated conformation for each membrane-bound simulation is shown in Figure 7. 

indistinguishable from each other. The $\mathrm{N}$-terminus sits $2 \AA$ lower in the membrane than the Cterminus (Table 2). Given that the termini were uncharged this is likely a property of the geometry of the peptide. In contrast to CgProt 2.3 simulations with charged termini and the hydrophobic $c a$ type backbone in which LS3 preferred the transmembrane configuration (Fosso-

332 Tande et al., 2017), the umbrella sampling PMF obtained for insertion of the neutral LS3

333 confirms that the interfacial conformation is the only preferred energy minimum in CgProt 2.4

334 (Fig. 8). The PMF at $z=0$ is $65 \mathrm{~kJ} / \mathrm{mol}$ higher in energy than the interfacial state found at a depth

335 of $z=1.2 \mathrm{~nm}$. When restrained to the center of the bilayer, LS3 adopts an unstable

336 transmembrane orientation in which the serine sidechains pull lipid head groups into the bilayer

337 interior. While phospholipid flip-flop has not been observed in pure membrane simulations with

338 CgProt, enhancement of lipid flip-flop by transmembrane hydrophilic residues is consistent with

339 recent experiments on endoplasmic reticulum peptide sequences (Nakao et al., 2016). The

340 pronounced stabilization of interfacial LS3 is in contrast to unrestrained simulations with the

341 Bond CG model (Bond et al., 2008; Hall et al., 2011), a variant of Martini, in which the

342 interfacial and transmembrane conformations were observed with $80 \%$ and $20 \%$ frequency,

343 respectively. In situ, the amphipathic LS3 peptide is only expected to adopt a transmembrane

344 orientation when residing in the membrane as part of a homo-oligomeric bundle, which serves as 345 an ion channel (Nguyen et al., 2013).

346 The PMF for the bilayer insertion of WALP23 reveals that the transmembrane

347 configuration centered near $z=0$ is preferred over the interfacial state in the vicinity of $z=0.9$

$348 \mathrm{~nm}$ by $15.5 \mathrm{~kJ} / \mathrm{mol}$ (Fig. 8). The interfacial state is a local energy minimum but has a minimal 1

$349 \mathrm{~kJ}$ barrier separating it from the transmembrane state. It is therefore not expected to be stable in 
350 unbiased molecular dynamics simulations. The stable insertion of neutral WALP is a

351 considerable improvement in $\operatorname{CgProt} 2.4$. The $24 \pm 5^{\circ}$ tilt angle obtained by fluorescence

352 spectroscopy (Holt et al., 2009) is within two standard deviations of the range observed in the

353 unrestrained simulation $\left(41 \pm 7^{\circ}\right)$. In contrast, the hydrophobic $c a$ type backbone employed in

354 previous versions causes uncharged WALP peptide termini to submerge in the hydrophobic tail

355 region of the bilayer (Fig. S1, Supplemental Information). The $93 \mathrm{~kJ} / \mathrm{mol}$ stabilization of

356 transmembrane WALP in the current version is comparable to the $146 \mathrm{~kJ} / \mathrm{mol}$ energy minimum

357 observed for the PLUM force field in a POPC bilayer (Bereau et al., 2015). In contrast,

358 transmembrane and interfacial WALP orientations have been observed with $80 \%$ and $20 \%$

359 frequency, respectively, in variants of the Martini model (Bereau \& Kremer, 2016; Hall et al., $3602011)$.

361 The previous $c a$ type hydrophobic backbone enabled the insertion of peptides to be

362 observed within $50 \mathrm{~ns}$ of unbiased molecular dynamics simulation. Optimizing the balance of

363 backbone and sidechain energetics in the current version, however, resulted in no membrane

364 insertion events being observed in $8 \mu$ s of simulations starting from the desorbed state. This

365 includes the $\mathrm{L}_{24}$ and (LA) 12 peptides, which were observed to adsorb onto the membrane surface

366 but not cross over to the other monolayer on the timescale of the simulation. This can be

367 explained by the presence of a $10 \mathrm{~kJ}$ barrier for the polar type backbone sites of non-alanine

368 residues to cross the center of the bilayer (Fig. 6). Additionally, the terminal lysines in the

369 peptides each have a $4 \mathrm{~kJ}$ barrier to penetrating the bilayer interface. These studies highlight the

370 need for either manual insertion of a protein into the bilayer or the use of biased sampling

371 methods.

\section{Conclusion}


373 The CgProt nonbond parameters have been refined to improve the permeation behavior for the

374 peptide backbone and amino acid sidechain analogs (Hu et al., 2014). The backbone unit has

375 been made less attractive for the bilayer by assigning the polar site type for non-alanine residues

376 and the apolar site type for alanine. Additional polar sites have been assigned for sidechains Arg,

377 Asp, Lys, Gln, Glu, Tyr, and Phe. The permeation of version 2.4 sidechains correlates well with

378 PMFs from atomistic simulation and experimental hydrophobicity scales based on cyclohexane-

379 to-water and octanol-to-water partition energies. Atomistic simulations with OPLS overestimate

380 the attraction of Gln, Arg and Lys for the ester region of the bilayer. The permeation of Asn and

381 Lys is much improved in CgProt 2.4 compared to v.1, while Gln and Arg experience a favorable

382 attraction similar to atomistic simulations. That Arg is more attractive to the membrane than Lys

383 in CgProt is consistent with the enhanced activity of arginine containing antimicrobial peptides

384 versus their lysine containing counterparts (Rice \& Wereszczynski, 2017). Note that three

385 sidechain assignments employed in v.2.3 are not recommended based on their permeation

386 behavior: the p.p.pos description of Lys, the ap-p-ap-p description of Trp, and the p-p description

387 of Asn. Previous simulations with v.2.3 revealed that the positive N-terminus has enhanced

388 permeation properties compared to the negative C-terminus (Fosso-Tande et al., 2017). The

389 enhanced membrane permeation of positive charges as opposed to negative site types is

390 supported by the CgProt PMF calculations.

391 Refining the CgProt force field by matching sidechain permeation profiles resulted in

392 improved simulations of membrane-associated peptides. While previous CgProt versions relied

393 on the use of terminal charges to anchor peptides and proteins in the membrane (Fosso-Tande et

394 al., 2017), the global energy minimum is now correctly obtained for the interfacial LS3 and

395 transmembrane WALP peptides. The improved backbone and sidechain descriptions in CgProt 
396

397

398

399

400

401

402

403

404

405

406

407

408

409

410

411

412

413

414

415

416

417

418

419

420

421

422

423

2.4 enable the simulation of diverse membrane protein systems and will serve to guide future model development efforts. The CgProt force field is an effective tool for studies of lipid-protein interactions and protein conformational change. To date protein-protein association has not been studied in CgProt. Refinements in amino acid hydrophobicity are likely key to reproducing experimentally observed dimer structures, which has proved a considerable challenge in CG models such as Martini (Javanainen et al., 2017). The CgProt tools and parameters needed for implementing molecular dynamics simulations in the Gromacs software package are included in the Supplemental Information, along with equilibrated bilayer coordinates.

\section{Acknowledgements}

R.D.H. thanks Jacob Fosso-Tande for valuable assistance.

\section{Figure Legends}

Figure 1. Site assignments in CgProt 2.4. Alpha carbons not involving alanine are reassigned the polar site type (sky blue). Other nonbond interaction types include positive (blue), negative (red), and apolar (gray). Asterisks denote new sidechain descriptions for version 2.4.

Figure 2. Partial lipid density profiles relative to the bilayer center $(z=0)$ for the $\mathbf{C G}$ DOPC simulation. Vertical gray bars divide the bilayer into four regions: hydrophobic tails, esters, lipid head groups, and solvent.

\section{Figure 3. PMF insertion profiles for mass centers of sidechain analogs relative to the} bilayer center. PMFs are set to zero in water. PMFs from atomistic simulations are compared in black (MacCallum et al., 2008). The bonding of CG sites is depicted for polar (p), apolar (ap), positive (pos) and negative (neg) types. Shorter bonds (.) were tested for Lys/Arg. Alternate sidechain descriptions shown in gray are not recommended. Various experimental data are plotted relative to zero for glycine. For sidechain analogs, the water-cyclohexane transfer energy is marked $c$ at $z=0$ and the water-octanol energy is marked $o$ at $z=1.3 \mathrm{~nm}$ (Radzicka \& Wolfenden, 1988). $\Delta G^{\mathrm{cor}}$ for the octanol solvation of a sidechain in a peptide occluded by moderate-sized neighbors is marked $w$ at $z=1.3 \mathrm{~nm}$ (Wimley et al., 1996). $\Delta G^{\mathrm{GXG}}$ for a fully 
424 exposed sidechain in GGXGG peptide is marked $*_{\mathrm{g}}$ at $1.3 \mathrm{~nm}$ for Leu, Val, Pro and Tyr. The 425 sidechain solvation energy for a WLXLL peptide at the POPC interface is marked $w$ at $z=1.7$ 426 nm (Wimley \& White, 1996).

427

Figure 4. Snapshot of p-p-pos Arg (cyan-cyan-blue spheres) restrained near the center of the bilayer. A bilayer defect solvates the CgProt sidechain similar to observations in atomistic MD (MacCallum et al., 2008). DOPC head groups are colored as blue (choline), red (phosphate) and green (glycerol) balls. Lipid tails are traced as sticks.

Figure 5. Linear regression of CgProt 2.4 sidechain solvation energies with experimental data. Single letter amino acid codes are plotted along with select 3-letter codes in parentheses. Water to cyclohexane (A) and water to octanol (B) transfer energies of unionized sidechain analogs (Radzicka \& Wolfenden, 1988) are correlated to PMF values at $z=0$ and $z=1.3 \mathrm{~nm}$, respectively. Wimley-White peptide residue transfer energies relative to glycine are correlated to PMF values at $z=1.3$ and $1.7 \mathrm{~nm}$ for octanol-water (C) and the POPC interface (D), respectively.

Figure 6. CgProt PMFs for one and two-residue backbone moieties. CgProt v.1 employed a unique alpha carbon site type (ca) as opposed to polar sites (p). Ca: The atomistic PMF for acetamide is drawn in black as an approximation for the glycyl $-\mathrm{CH}_{2} \mathrm{CONH}$ - unit (MacCallum et $a l ., 2008)$. Cyclohexane and octanol measurements for acetamide are labeled $c$ and $o$ at 0 and 1.3 $\mathrm{nm}$, respectively. Whole-residue Wimley-White measurements of glycine are labeled $w$ at 1.3 and $1.7 \mathrm{~nm}$. Co-Co: Twice the atomistic PMF for acetamide is shown (black line). $2 w$ corresponds to twice the Wimley-White value for glycine at 1.3 and $1.7 \mathrm{~nm}$. The water-octanol transfer energy of the Gly dipeptide is labeled d* at $1.3 \mathrm{~nm}$ (Fauchere \& Pliska, 1983). Ala: The atomistic PMF of methane $\left(\mathrm{CH}_{4}\right)$ is shifted to account for glycine's $1.15 \mathrm{kcal}$ octanol solvation penalty (black). Whole-residue Wimley-White values for alanine are labeled $w$. The PMF of a single CgProt apolar site (ap) is shown in aqua.

\section{Figure 7. Equilibrated peptide orientations in $\mu$ s membrane-bound simulations. The LS3} peptide inserts in an interfacial orientation, while $\mathrm{L}_{24},(\mathrm{LA})_{12}$, and WALP23 remain in a transmembrane orientation. The surface of lipid head groups is shown in transparent blue (choline), red (phosphate), green (glycerol) and white (esters). The C $\alpha$ backbone is traced in cyan with neutral N- and C-termini labeled blue and red, respectively. Spheres are colored for polar sites belonging to serine (gold) and tryptophan (green) sidechains and for the positive charge of lysine (cream). 
461 Figure 8. Peptide insertion PMFs. Independent PMFs were obtained for the permeation of LS3 462 and WALP23 in both leaflets of the bilayer (dashed lines). The symmetrized PMF (solid line) 463 reveals a global energy minimum for both interfacial LS3 and transmembrane WALP23.

464

References

466

467

468

469

470

471

472

473

474

475

476

477

478

479

480

481

482

483

484

485

486

487

488

489

490

491

492

493

494

495

496

497

498

499

500

501

Arnarez C, Uusitalo JJ, Masman MF, Ingolfsson HI, de Jong DH, Melo MN, Periole X, de Vries AH, Marrink SJ. 2015. Dry Martini, a coarse-grained force field for lipid membrane simulations with implicit solvent. Journal of Chemical Theory and Computation 11:260-275 DOI 10.1021/ct500477k.

Bennett WF, Tieleman DP. 2013. Computer simulations of lipid membrane domains. Biochimica et Biophysica Acta - Biomembranes 1828:1765-1776 DOI 10.1016/j.bbamem.2013.03.004.

Bereau T, Bennett WF, Pfaendtner J, Deserno M, Karttunen M. 2015. Folding and insertion thermodynamics of the transmembrane WALP peptide. Journal of Chemical Physics 143:243127 DOI 10.1063/1.4935487.

Bereau T, Kremer K. 2016. Protein-backbone thermodynamics across the membrane interface. Journal of Physical Chemistry B 120:6391-6400 DOI 10.1021/acs.jpcb.6b03682.

Bereau T, Wang ZJ, Deserno M. 2014. More than the sum of its parts: Coarse-grained peptide-lipid interactions from a simple cross-parametrization. Journal of Chemical Physics 140:115101 DOI 10.1063/1.4867465.

Bond PJ, Holyoake J, Ivetac A, Khalid S, Sansom MS. 2007. Coarse-grained molecular dynamics simulations of membrane proteins and peptides. Journal of Structural Biology 157:593-605 DOI 10.1016/j.jsb.2006.10.004.

Bond PJ, Wee CL, Sansom MS. 2008. Coarse-grained molecular dynamics simulations of the energetics of helix insertion into a lipid bilayer. Biochemistry 47:11321-11331 DOI 10.1021/bi800642m.

Cerutti DS, Duke R, Freddolino PL, Fan H, Lybrand TP. 2008. Vulnerability in popular molecular dynamics packages concerning Langevin and Andersen dynamics. Journal of Chemical Theory and Computation 4:1669-1680 DOI 10.1021/ct8002173.

de Jong DH, Singh G, Bennett WFD, Arnarez C, Wassenaar TA, Schafer LV, Periole X, Tieleman DP, Marrink SJ. 2013. Improved parameters for the Martini coarse-grained protein force field. Journal of Chemical Theory and Computation 9:687-697 DOI 10.1021/ct300646g.

Fauchere J, Pliska V. 1983. Hydrophobic parameters II of amino-acid side chains from the partitioning of N-acetyl-amino-acid amides. European Journal of Medicinal Chemistry Chimica Therapeutica 18:369-375 DOI

Fosso-Tande J, Black C, Aller SG, Lu L, Hills Jr RD. 2017. Simulation of lipid-protein interactions with the CgProt force field. AIMS Molecular Science 4:352-369 DOI 10.3934/molsci.2017.3.352.

Ganesan SJ, Matysiak S. 2014. Role of backbone dipole interactions in the formation of secondary and supersecondary structures of proteins. Journal of Chemical Theory and Computation 10:2569-2576 DOI 10.1021/ct401087a. 
502

503

504

505

506

507

508

509

510

511

512

513

514

515

516

517

518

519

520

521

522

523

524

525

526

527

528

529

530

531

532

533

534

535

536

537

538

539

540

541

542

543

544

545
Genheden S, Essex JW. 2015. A simple and transferable all-atom/coarse-grained hybrid model to study membrane processes. Journal of Chemical Theory and Computation 11:47494759 DOI 10.1021/acs.jctc.5b00469.

Gleason NJ, Vostrikov VV, Greathouse DV, Koeppe II RE. 2013. Buried lysine, but not arginine, titrates and alters transmembrane helix tilt. Proceedings of the National Academy of Sciences, USA 110:1692-1695 DOI 10.1073/pnas.1215400110.

Gumbart J, Roux B. 2012. Determination of membrane-insertion free energies by molecular dynamics simulations. Biophysical Journal 102:795-801 DOI 10.1016/j.bpj.2012.01.021.

Hall BA, Chetwynd AP, Sansom MS. 2011. Exploring peptide-membrane interactions with coarse-grained MD simulations. Biophysical Journal 100:1940-1948 DOI 10.1016/j.bpj.2011.02.041.

Han W, Wan CK, Wu YD. 2008. Toward a coarse-grained protein model coupled with a coarse-grained solvent model: Solvation free energies of amino acid side chains. Journal of Chemical Theory and Computation 4:1891-1901 DOI 10.1021/ct800184c.

Hedger G, Sansom MS. 2016. Lipid interaction sites on channels, transporters and receptors: Recent insights from molecular dynamics simulations. Biochimica et Biophysica Acta Biomembranes 1858:2390-2400 DOI 10.1016/j.bbamem.2016.02.037.

Hills Jr RD. 2014. Balancing bond, nonbond, and Go-like terms in coarse grain simulations of conformational dynamics. Methods in Molecular Biology 1084:123-140 DOI 10.1007/978-162703-658-0_7.

Hills Jr RD, Lu L, Voth GA. 2010. Multiscale coarse-graining of the protein energy landscape. PLOS Computational Biology 6:e1000827 DOI 10.1371/journal.pcbi.1000827.

Hills Jr RD, McGlinchey N. 2016. Model parameters for simulation of physiological lipids. Journal of Computational Chemistry 37:1112-1118 DOI 10.1002/jcc.24324.

Holt A, Koehorst RB, Rutters-Meijneke T, Gelb MH, Rijkers DT, Hemminga MA, Killian JA. 2009. Tilt and rotation angles of a transmembrane model peptide as studied by fluorescence spectroscopy. Biophysical Journal 97:2258-2266 DOI 10.1016/j.bpj.2009.07.042.

Hu Y, Sinha SK, Patel S. 2014. Reconciling structural and thermodynamic predictions using all-atom and coarse-grain force fields: The case of charged oligo-arginine translocation into DMPC bilayers. Journal of Physical Chemistry B 118:11973-11992 DOI 10.1021/jp504853t.

Hub JS, de Groot BL, van der Spoel D. 2013. g_wham: A free weighted histogram analysis implementation including robust error and autocorrelation estimates. Journal of Chemical Theory and Computation 6:3713-3720 DOI 10.1021/ct300646g.

Javanainen M, Martinez-Seara H, Vattulainen I. 2017. Excessive aggregation of membrane proteins in the Martini model. PLOS ONE 12:e0187936 DOI 10.1371/journal.pone.0187936.

Johansson AC, Lindahl E. 2008. Position-resolved free energy of solvation for amino acids in lipid membranes from molecular dynamics simulations. Proteins: Structure, Function, and Bioinformatics 70:1332-1344 DOI 10.1002/prot.21629.

Kar P, Gopal SM, Cheng YM, Panahi A, Feig M. 2014. Transferring the PRIMO coarsegrained force field to the membrane environment: Simulations of membrane proteins and helixhelix association. Journal of Chemical Theory and Computation 10:3459-3472 DOI $10.1021 /$ ct500443v.

Karanicolas J, Brooks III CL. 2002. The origins of asymmetry in the folding transition states of protein L and protein G. Protein Science 11:2351-2361 DOI 10.1110/ps.0205402. 
546

547

548

549

550

551

552

553

554

555

556

557

558

559

560

561

562

563

564

565

566

567

568

569

570

571

572

573

574

575

576

577

578

579

580

581

582

583

584

585

586

587

588

589

590

Kim T, Im W. 2010. Revisiting hydrophobic mismatch with free energy simulation studies of transmembrane helix tilt and rotation. Biophysical Journal 99:175-183 DOI 10.1016/j.bpj.2010.04.015.

Kmiecik S, Gront D, Kolinski M, Wieteska L, Dawid AE, Kolinski A. 2016. Coarsegrained protein models and their applications. Chemical Reviews 116:7898-7936 DOI 10.1021/acs.chemrev.6b00163.

Lear JD, Wasserman ZR, DeGrado WF. 1988. Synthetic amphiphilic peptide models for protein ion channels. Science 240:1177-1181 DOI 10.1126/science.2453923.

Liu F, Lewis RN, Hodges RS, McElhaney RN. 2004. Effect of variations in the structure of a polyleucine-based alpha-helical transmembrane peptide on its interaction with phosphatidylethanolamine bilayers. Biophysical Journal 87:2470-2482 DOI 10.1529/biophysj.104.046342.

Lu L, Voth GA. 2009. Systematic coarse-graining of a multicomponent lipid bilayer. Journal of Physical Chemistry B 113:1501-1510 DOI 10.1021/jp809604k.

Ma H, Irudayanathan FJ, Jiang W, Nangia S. 2015. Simulating gram-negative bacterial outer membrane: A coarse grain model. Journal of Physical Chemistry B 119:14668-14682 DOI 10.1021/acs.jpcb.5b07122.

MacCallum JL, Bennett WF, Tieleman DP. 2008. Distribution of amino acids in a lipid bilayer from computer simulations. Biophysical Journal 94:3393-3404 DOI 10.1529/biophysj.107.112805.

MacCallum JL, Bennett WF, Tieleman DP. 2011. Transfer of arginine into lipid bilayers is nonadditive. Biophysical Journal 101:110-117 DOI 10.1016/j.bpj.2011.05.038.

MacCallum JL, Tieleman DP. 2011. Hydrophobicity scales: A thermodynamic looking glass into lipid-protein interactions. Trends in Biochemical Sciences 36:653-662 DOI 10.1016/j.tibs.2011.08.003.

Marrink SJ, Tieleman DP. 2013. Perspective on the Martini model. Chemical Society Reviews 42:6801-6822 DOI 10.1039/c3cs60093a.

Nakao H, Ikeda K, Ishihama Y, Nakano M. 2016. Membrane-spanning sequences in endoplasmic reticulum proteins promote phospholipid flip-flop. Biophysical Journal 110:26892697 DOI 10.1016/j.bpj.2016.05.023.

Neale C, Pomes R. 2016. Sampling errors in free energy simulations of small molecules in lipid bilayers. Biochimica et Biophysica Acta - Biomembranes 1858:2539-2548 DOI 10.1016/j.bbamem.2016.03.006.

Nguyen TH, Liu Z, Moore PB. 2013. Molecular dynamics simulations of homo-oligomeric bundles embedded within a lipid bilayer. Biophysical Journal 105:1569-1580 DOI 10.1016/j.bpj.2013.07.053.

Nitschke N, Atkovska K, Hub JS. 2016. Accelerating potential of mean force calculations for lipid membrane permeation: System size, reaction coordinate, solute-solute distance, and cutoffs. Journal of Chemical Physics 145:125101 DOI 10.1063/1.4963192.

Noid WG, Chu JW, Ayton GS, Krishna V, Izvekov S, Voth GA, Das A, Andersen HC. 2008. The multiscale coarse-graining method. I. A rigorous bridge between atomistic and coarsegrained models. Journal of Chemical Physics 128:244114 DOI 10.1063/1.2938860.

Noid WG, Chu JW, Ayton GS, Voth GA. 2007. Multiscale coarse-graining and structural correlations: Connections to liquid-state theory. Journal of Physical Chemistry B 111:4116-4127 DOI 10.1021/jp068549t. 
600

601

602

603

604

605

606

607

608

609

610

611

612

613

614

615

616

617

618

619

620

621

622

623

624

625

626

627

628

629

630

631

632

633

634

635

636

Pall S, Abraham MJ, Kutzner C, Hess B, Lindahl E. 2015. Tackling exascale software challenges in molecular dynamics simulations with GROMACS. Solving Software Challenges for Exascale EASC 2014 Lecture Notes in Computer Science 8759:3-27 DOI 10.1007/978-3319-15976-8_1.

Pogorelov TV, Vermaas JV, Arcario MJ, Tajkhorshid E. 2014. Partitioning of amino acids into a model membrane: Capturing the interface. Journal of Physical Chemistry B 118:14811492 DOI 10.1021/jp4089113.

Poyry S, Vattulainen I. 2016. Role of charged lipids in membrane structures: Insight given by simulations. Biochimica et Biophysica Acta - Biomembranes 1858:2322-2333 DOI 10.1016/j.bbamem.2016.03.016.

Pulawski W, Jamroz M, Kolinski M, Kolinski A, Kmiecik S. 2016. Coarse-grained simulations of membrane insertion and folding of small helical proteins using the CABS model. Journal of Chemical Information and Modeling 56:2207-2215 DOI 10.1021/acs.jcim.6b00350.

Radzicka A, Wolfenden R. 1988. Comparing the polarities of the amino acids: Side-chain distribution coefficients between the vapor phase, cyclohexane, 1-ocatnol, and neutral aqueous solution. Biochemistry 27:1664-1670 DOI 10.1021/bi00405a042.

Rice A, Wereszczynski J. 2017. Probing the disparate effects of arginine and lysine residues on antimicrobial peptide/bilayer association. Biochimica et Biophysica Acta - Biomembranes 1859:1941-1950 DOI 10.1016/j.bbamem.2017.06.002.

Sandoval-Perez A, Pluhackova K, Bockmann RA. 2017. Critical comparison of biomembrane force fields: Protein-lipid interactions at the membrane interface. Journal of Chemical Theory and Computation 13:2310-2321 DOI 10.1021/acs.jctc.7b00001.

Singh G, Tieleman DP. 2011. Using the Wimley-White hydrophobicity scale as a direct quantitative test of force fields: The MARTINI coarse-grained model. Journal of Chemical Theory and Computation 7:2316-2324 DOI 10.1021/ct2002623.

Sun D, Forsman J, Woodward CE. 2015. Evaluating force fields for the computational prediction of ionized arginine and lysine side-chains partitioning into lipid bilayers and octanol. Journal of Chemical Theory and Computation 11:1775-1791 DOI 10.1021/ct501063a.

Van Oosten B, Harroun TA. 2016. A MARTINI extension for Pseudomonas aeruginosa PAO1 lipopolysaccharide. Journal of Molecular Graphics \& Modelling 63:125-133 DOI 10.1016/j.jmgm.2015.12.002.

Venable RM, Ingolfsson HI, Lerner MG, Perrin BS, Jr., Camley BA, Marrink SJ, Brown FLH, Pastor RW. 2017. Lipid and peptide diffusion in bilayers: The Saffman-Delbruck model and periodic boundary conditions. Journal of Physical Chemistry B 121:3443-3457 DOI 10.1021/acs.jpcb.6b09111.

Vorobyov I, Kim I, Chu ZT, Warshel A. 2016. Refining the treatment of membrane proteins by coarse-grained models. Proteins: Structure, Function, and Bioinformatics 84:92-117 DOI 10.1002/prot.24958.

Ward AB, Guvench O, Hills Jr RD. 2012. Coarse grain lipid-protein molecular interactions and diffusion with MsbA flippase. Proteins: Structure, Function, and Bioinformatics 80:21782190 DOI 10.1002/prot.24108.

Ward MD, Nangia S, May ER. 2017. Evaluation of the hybrid resolution PACE model for the study of folding, insertion, and pore formation of membrane associated peptides. Journal of Computational Chemistry 38:1462-1471 DOI 10.1002/jcc.24694.

White SH, Wimley WC. 1998. Hydrophobic interactions of peptides with membrane interfaces. Biochimica et Biophysica Acta 1376:339-352 DOI 10.1016/S0304-4157(98)00021-5. 
637 Wimley WC, Creamer TP, White SH. 1996. Solvation energies of amino acid side chains 638 and backbone in a family of host-guest pentapeptides. Biochemistry 35:5109-5124 DOI $63910.1021 /$ bi9600153.

640 Wimley WC, White SH. 1996. Experimentally determined hydrophobicity scale for proteins 641 at membrane interfaces. Nature Structural Biology 3:842-848 DOI 10.1038/nsb1096-842.

642 Zhang YP, Lewis RN, Henry GD, Sykes BD, Hodges RS, McElhaney RN. 1995. Peptide 643 models of helical hydrophobic transmembrane segments of membrane proteins. 1. Studies of the 644 conformation, intrabilayer orientation, and amide hydrogen exchangeability of Ac-K2-(LA)12645 K2-amide. Biochemistry 34:2348-2361 DOI 10.1021/bi00007a031.

646

647 
Figure 1 (on next page)

Site assignments in CgProt 2.4 .

Alpha carbons not involving alanine are reassigned the polar site type (sky blue). Other nonbond interaction types include positive (blue), negative (red), and apolar (gray). Asterisks denote new sidechain descriptions for version 2.4. 


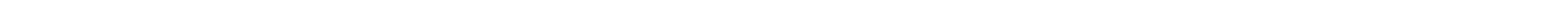




\section{Figure 2 (on next page)}

Partial lipid density profiles relative to the bilayer center $(z=0)$ for the CG DOPC simulation.

Vertical gray bars divide the bilayer into four regions: hydrophobic tails, esters, lipid head groups, and solvent. 


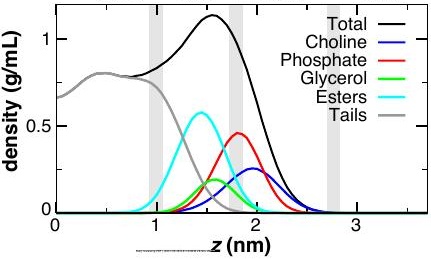




\section{Figure 3 (on next page)}

PMF insertion profiles for mass centers of sidechain analogs relative to the bilayer center.

PMFs are set to zero in water. PMFs from atomistic simulations are compared in black ( MacCallum et al., 2008 ). The bonding of CG sites is depicted for polar (p), apolar (ap), positive (pos) and negative (neg) types. Shorter bonds (.) were tested for Lys/Arg. Alternate sidechain descriptions shown in gray are not recommended. Various experimental data are plotted relative to zero for glycine. For sidechain analogs, the water-cyclohexane transfer energy is marked $c$ at $z=0$ and the water-octanol energy is marked $o$ at $z=1.3 \mathrm{~nm}$ ( Radzicka \& Wolfenden, 1988 ) . $\Delta G^{\text {cor }}$ for the octanol solvation of a sidechain in a peptide occluded by moderate-sized neighbors is marked $w$ at $z=1.3 \mathrm{~nm}$ (Wimley et al., 1996 ) . $\Delta G^{\mathrm{GXG}}$ for a fully exposed sidechain in GGXGG peptide is marked ${ }^{*} \mathrm{~g}$ at $1.3 \mathrm{~nm}$ for Leu, Val, Pro and Tyr. The sidechain solvation energy for a WLXLL peptide at the POPC interface is marked $w$ at $z=1.7 \mathrm{~nm}$ ( Wimley \& White, 1996 ). 


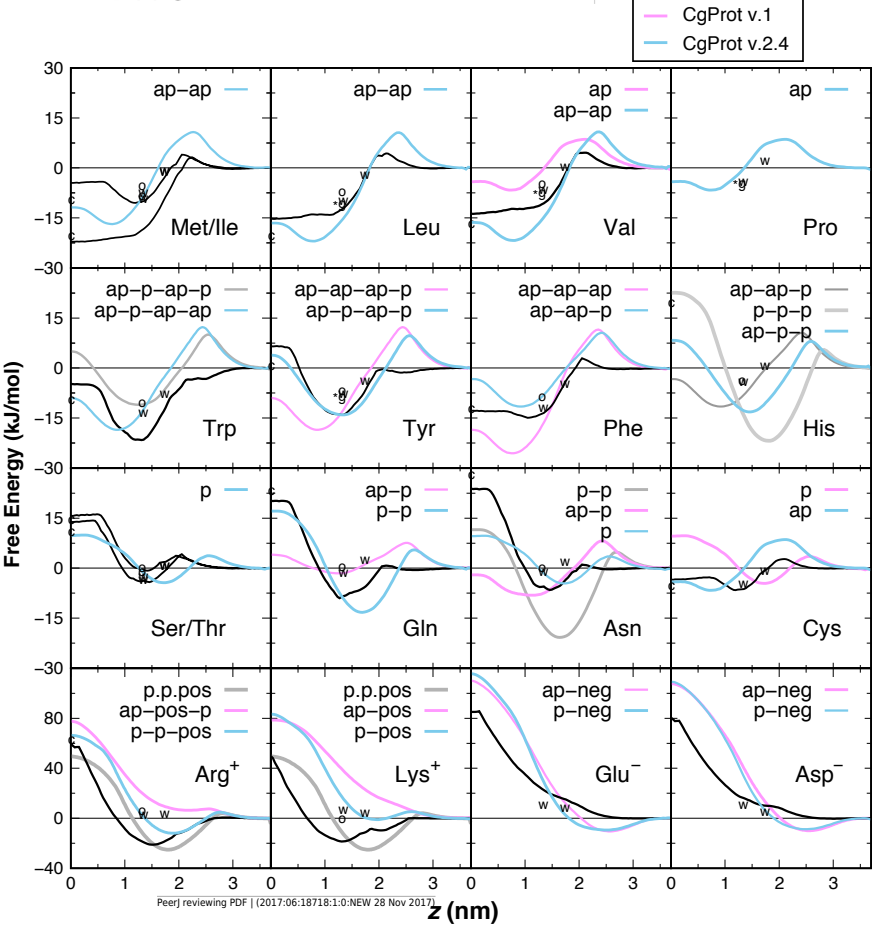




\section{Figure 4}

Snapshot of p-p-pos Arg (cyan-cyan-blue spheres) restrained near the center of the bilayer.

A bilayer defect solvates the CgProt sidechain similar to observations in atomistic MD ( MacCallum et al., 2008 ) . DOPC head groups are colored as blue (choline), red (phosphate) and green (glycerol) balls. Lipid tails are traced as sticks.

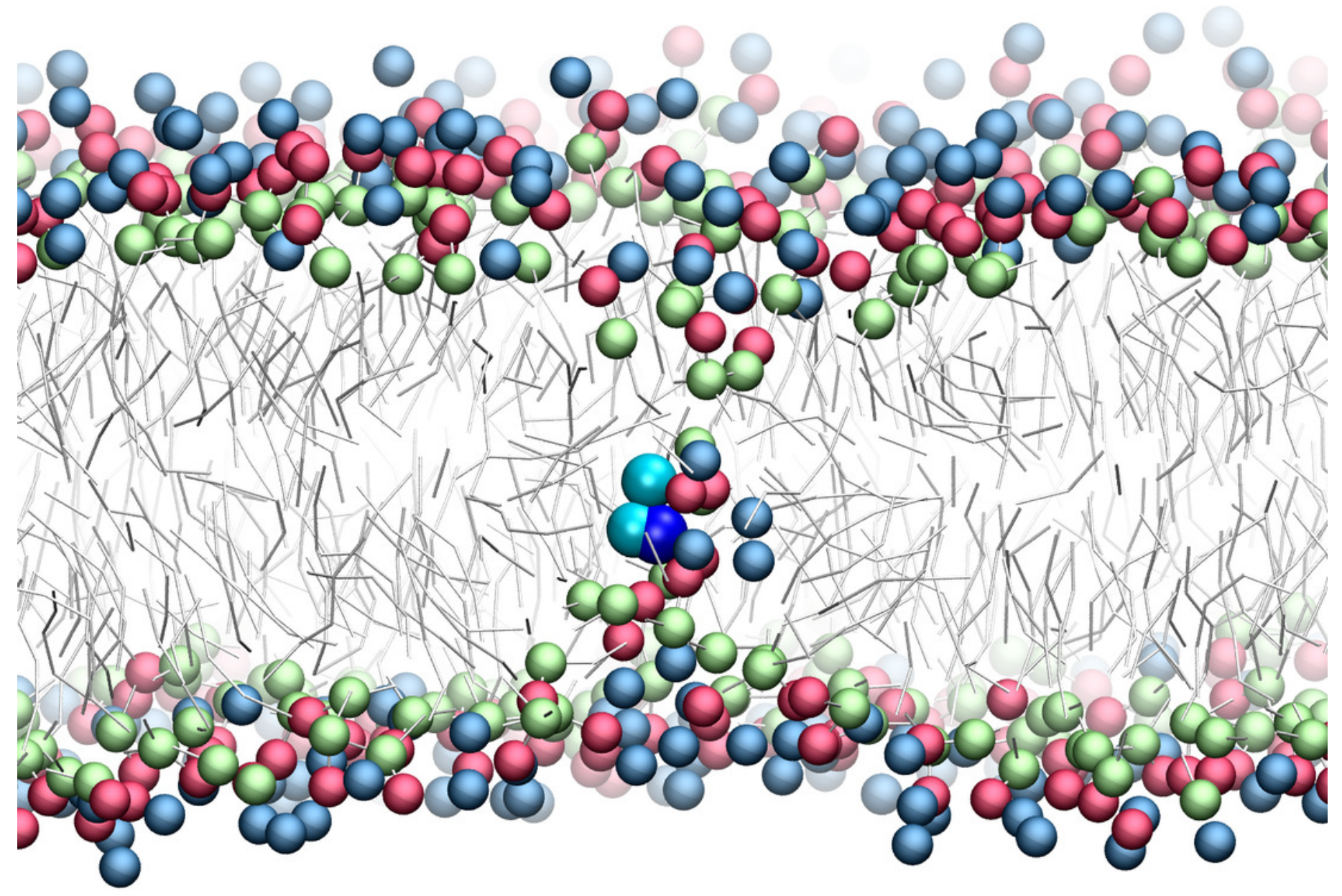


Figure $\mathbf{5}$ (on next page)

Linear regression of CgProt 2.4 sidechain solvation energies with experimental data.

Single letter amino acid codes are plotted along with select 3-letter codes in parentheses. Water to cyclohexane (A) and water to octanol (B) transfer energies of unionized sidechain analogs ( Radzicka \& Wolfenden, 1988 ) are correlated to PMF values at $z=0$ and $z=1.3$ $\mathrm{nm}$, respectively. Wimley-White peptide residue transfer energies relative to glycine are correlated to PMF values at $z=1.3$ and $1.7 \mathrm{~nm}$ for octanol-water (C) and the POPC interface (D), respectively. 

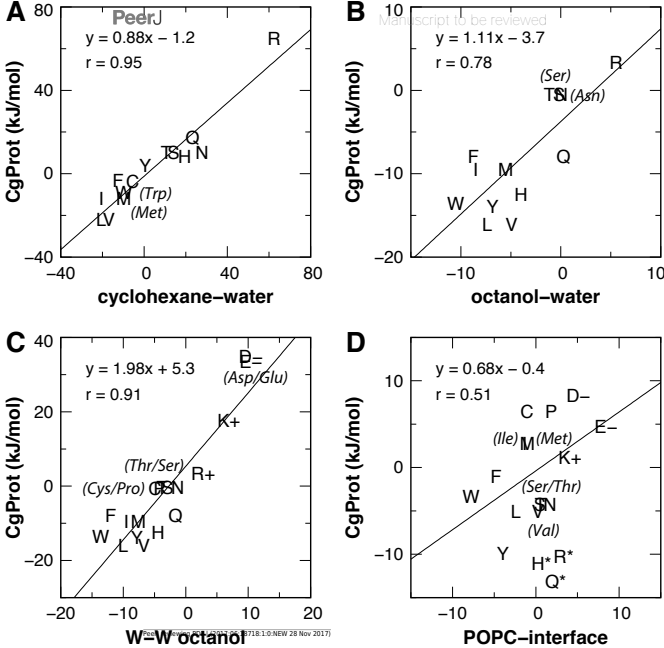


\section{Figure 6 (on next page)}

CgProt PMFs for one and two-residue backbone moieties.

CgProt v.1 employed a unique alpha carbon site type (ca) as opposed to polar sites (p). $\mathbf{C} \boldsymbol{\alpha}$ : The atomistic PMF for acetamide is drawn in black as an approximation for the glycyl $-\mathrm{CH}_{2} \mathrm{CONH}$ - unit ( MacCallum et al., 2008 ) . Cyclohexane and octanol measurements for acetamide are labeled $c$ and $o$ at 0 and $1.3 \mathrm{~nm}$, respectively. Whole-residue Wimley-White measurements of glycine are labeled $w$ at 1.3 and $1.7 \mathrm{~nm}$. $\mathbf{C} \boldsymbol{\alpha}-\mathbf{C} \boldsymbol{\alpha}$ : Twice the atomistic PMF for acetamide is shown (black line). $2 w$ corresponds to twice the Wimley-White value for glycine at 1.3 and $1.7 \mathrm{~nm}$. The water-octanol transfer energy of the Gly dipeptide is labeled d* at $1.3 \mathrm{~nm}$ ( Fauchere \& Pliska, 1983 ). Ala: The atomistic PMF of methane $\left(\mathrm{CH}_{4}\right)$ is shifted to account for glycine's $1.15 \mathrm{kcal}$ octanol solvation penalty (black). Whole-residue WimleyWhite values for alanine are labeled $w$. The PMF of a single CgProt apolar site (ap) is shown in aqua. 


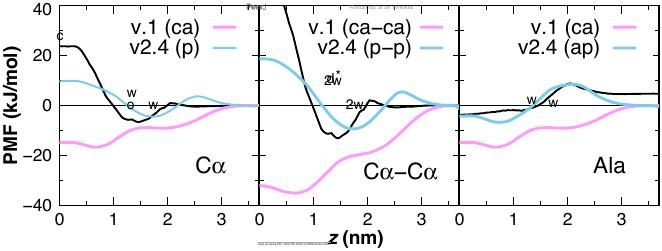




\section{Figure 7}

Equilibrated peptide orientations in $\mu$ s membrane-bound simulations.

The LS3 peptide inserts in an interfacial orientation, while $\mathrm{L}_{24}(\mathrm{LA})_{12}$, and WALP23 remain in a transmembrane orientation. The surface of lipid head groups is shown in transparent blue (choline), red (phosphate), green (glycerol) and white (esters). The C $\alpha$ backbone is traced in cyan with neutral $\mathrm{N}$ - and C-termini labeled blue and red, respectively. Spheres are colored for polar sites belonging to serine (gold) and tryptophan (green) sidechains and for the positive charge of lysine (cream). 


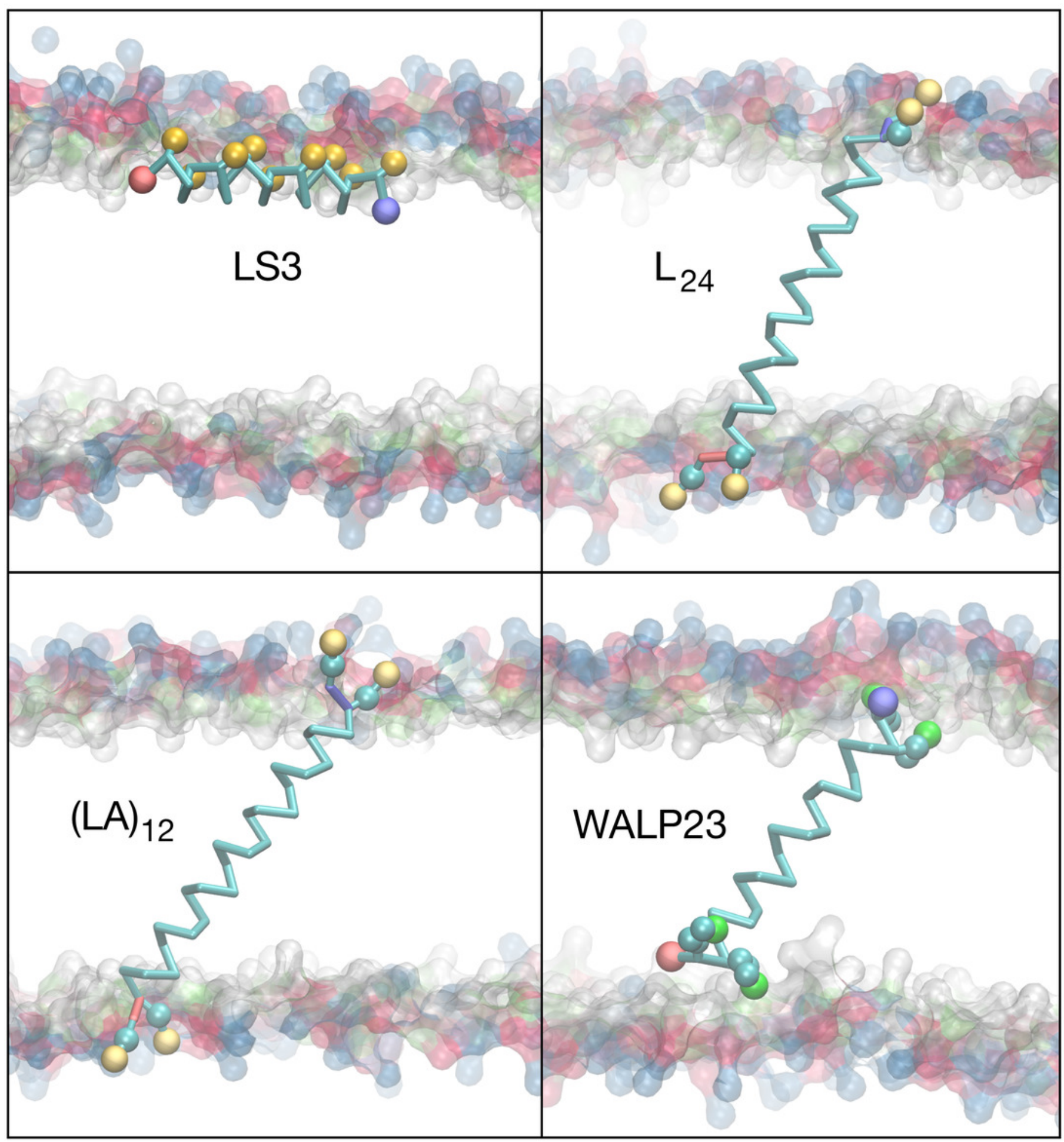




\section{Figure 8 (on next page)}

\section{Peptide insertion PMFs.}

Independent PMFs were obtained for the permeation of LS3 and WALP23 in both leaflets of the bilayer (dashed lines). The symmetrized PMF (solid line) reveals a global energy minimum for both interfacial LS3 and transmembrane WALP23. 

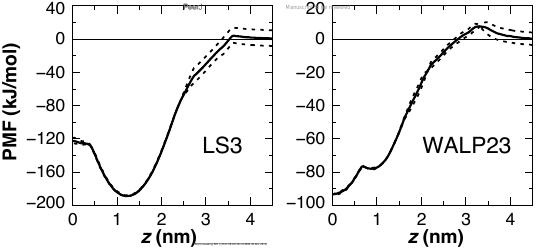


\section{Table $\mathbf{1}$ (on next page)}

MacCallum et al. and CG PMFs versus experiment

${ }^{\mathrm{a}} z=0 \mathrm{~nm} ;{ }^{\mathrm{b}} z=1.3 \mathrm{~nm} ;{ }^{\mathrm{c}}$ Excludes outliers Arg and Lys; ${ }^{\mathrm{d}} z=1.7 \mathrm{~nm}$; ${ }^{\mathrm{e}}$ Excludes outliers His, Arg and Gln. 
1 Table 1. MacCallum et al. and CG PMFs versus experiment

\begin{tabular}{ll|ccc}
\hline$x$ : Experiment $(\Delta G)$ & $y:$ PMF & $\begin{array}{l}\text { Pearson } \\
\text { correlation, } \mathrm{r}\end{array}$ & Slope & $\begin{array}{l}\text { Intercept } \\
(\mathrm{kJ} / \mathrm{mol})\end{array}$ \\
\hline Cyclohexane-water & Atomistic $^{\mathrm{a}}$ & 0.98 & 0.89 & 1.3 \\
Cyclohexane-water & CgProt $^{\mathrm{a}}$ & 0.95 & 0.88 & -1.2 \\
\hline Octanol-water & Atomistic $^{\mathrm{b}}$ & $0.92^{\mathrm{c}}$ & 1.6 & -2.6 \\
Octanol-water & CgProt $^{\mathrm{b}}$ & 0.78 & 1.1 & -3.7 \\
\hline $\mathrm{W}-\mathrm{W}$ octanol $\left(\Delta \mathrm{G}^{\mathrm{cor}}\right)$ & Atomistic $^{\mathrm{b}}$ & $0.95^{\mathrm{c}}$ & 1.6 & 0.8 \\
$\mathrm{~W}-\mathrm{W}$ octanol $\left(\Delta \mathrm{G}^{\mathrm{cor}}\right)$ & CgProt $^{\mathrm{b}}$ & 0.91 & 2.0 & 5.3 \\
\hline $\mathrm{W}-\mathrm{W}$ POPC interface & Atomistic $^{\mathrm{d}}$ & $0.86^{\mathrm{c}}$ & 1.6 & -1.6 \\
$\mathrm{~W}-\mathrm{W}$ POPC interface & CgProt $^{\mathrm{d}}$ & $0.51^{\mathrm{e}}$ & 0.68 & -0.4 \\
\hline
\end{tabular}

$2 \mathrm{a}_{z}=0 \mathrm{~nm}$

$3 \mathrm{~b}_{z}=1.3 \mathrm{~nm}$

$4{ }^{c}$ Excludes outliers Arg and Lys.

$5 d_{z}=1.7 \mathrm{~nm}$

$6{ }^{e}$ Excludes outliers His, Arg and Gln.

7 


\section{Table 2 (on next page)}

Peptide orientation during $1-2 \mu \mathrm{s}$ of simulation time

aMean angle \pm standard deviation of helix axis with respect to bilayer normal. bMean displacement of helix center of mass from bilayer center. 'Mean distance of terminal C $\alpha$ from bilayer center. 
1 Table 2. Peptide orientation during $1-2 \mu$ s of simulation time

\begin{tabular}{ll|cccc}
\hline Peptide & Starting state & $\begin{array}{c}\text { Helix tilt } \\
\left({ }^{\circ}\right)\end{array}$ & $\begin{array}{c}\mathrm{Z}_{\text {center }}{ }^{\mathrm{b}} \\
(\AA)\end{array}$ & $\mathrm{Z}_{\mathrm{N} \text {-term }}{ }^{\mathrm{c}}(\AA)$ & $\mathrm{Z}_{\mathrm{C} \text {-term }}{ }^{\mathrm{c}}(\AA)$ \\
\hline $\mathrm{LS} 3$ & desorbed & $85.1 \pm 5.5$ & $12.4 \pm 1.1$ & $11.4 \pm 1.7$ & $13.4 \pm 1.8$ \\
$\mathrm{LS} 3$ & interfacial & $84.6 \pm 5.4$ & $12.2 \pm 1.2$ & $11.0 \pm 1.7$ & $13.4 \pm 1.8$ \\
\hline $\mathrm{L}_{24}$ & desorbed & $85.4 \pm 3.7$ & $19.6 \pm 1.5$ & $19.0 \pm 1.5$ & $18.4 \pm 1.5$ \\
$\mathrm{~L}_{24}$ & transmembrane & $32.5 \pm 6.2$ & $-0.1 \pm 0.2$ & $16.8 \pm 1.8$ & $-18.2 \pm 2.0$ \\
\hline$(\mathrm{LA})_{12}$ & desorbed & $88.9 \pm 3.8$ & $13.6 \pm 1.1$ & $14.1 \pm 1.4$ & $13.8 \pm 1.5$ \\
$(\mathrm{LA})_{12}$ & transmembrane & $35.4 \pm 5.4$ & $0.7 \pm 1.3$ & $17.5 \pm 1.4$ & $-17.0 \pm 1.7$ \\
\hline WALP23 & desorbed & - & - & - & - \\
WALP23 & transmembrane & $41.0 \pm 7.3$ & $0.9 \pm 1.5$ & $13.3 \pm 1.8$ & $-10.2 \pm 2.5$ \\
\hline
\end{tabular}

2 a Mean angle \pm standard deviation of helix axis with respect to bilayer normal.

3 bMean displacement of helix center of mass from bilayer center.

$4{ }^{\mathrm{c}}$ Mean distance of terminal $\mathrm{C} \alpha$ from bilayer center. 